\title{
La survie des partis politiques et le militantisme transhumant et errant au Cameroun
}

\author{
Ismaila Datidjo \\ Valeri Duplexe Yotedje \\ Faculté des Lettres et Sciences Humaines, \\ Université de Dschang, Cameroun \\ Armand Tchinenba \\ Faculté des Sciences Juridiques et Politiques, \\ Université de Dschang, Cameroun
}

Doi:10.19044/esj.2021.v17n16p95

Submitted: 08 November 2020

Accepted: 11 May 2021

Published: 31 May 2021
Copyright 2021 Author(s)

Under Creative Commons BY-NC-ND

4.0 OPEN ACCESS

Cite As:

Datidjo I., Yotedje V.D. \& Tchinenba A. (2021). La survie des partis politiques et le militantisme transhumant et errant au Cameroun. European Scientific Journal, ESJ, 17(16), 95. https://doi.org/10.19044/esj.2021.v17n16p95

\section{Resume}

Au cours des trois (03) décennies qui ont suivi le retour du Cameroun à la démocratie plurielle en 1990, l'arène politique enregistre une multitude d'acteurs et d'organisations avec diverses opinions. De nos jours, plus de trois cent (300), formations politiques ont vu le jour avec différentes idéologies proposées aux citoyens. La loi ${ }^{\circ}$ 90/053/ du 19 décembre 1990 portant sur la liberté d'association, soutenue par celle $\mathrm{n}^{\circ}$ 90/056 du 19 Décembre 1990 portant sur la création des partis politiques a favorisé le multipartisme et le développement idéologique au Cameroun. Ainsi, il s'est développé des comportements de nomadismes ou de transhumances politiques chez beaucoup d'acteurs de la scène politique camerounaise. Cet article qui se veut une réflexion sur l'expérience démocratique des camerounais dans le contexte du multipartisme politique, rend compte de la façon selon laquelle des citoyens militent dans plusieurs formations politiques d'une manière cyclique le plus souvent ou continue. C'est en ces deux types de comportements que consistent respectivement le militantisme transhumant et celui errant. Ils remettent en question la survie sur l'arène politique de certaines forces politiques, les plus faibles, c'est-à-dire celles les moins adaptées au jeu électoral au profit de celles les plus puissantes, donc celles qui participent aux compétitions politiques et 
de surcroît, obtiennent le plus de suffrages. Ces dernières s'adaptent en récupérant les militants et sympathisants des organisations politiques adverses. Dans une approche analytique fondée sur la stratégie des acteurs du jeu politique impliqués dans le militantisme transhumant et errant, ce à partir des données du terrain et celles secondaires issues des résultats des élections jusque-là organisées, il rapporte le mécanisme par lequel certains partis politiques sont actifs, alors que d'autres ne le sont pas, ils sont plutôt absents des compétions, dirait-on, ils auraient cessé d'exister en tant que entités.

Mot clés : Militantisme transhumant, militantisme errant, partis politiques, démocratie

\title{
The Survival of Political Parties and the Transhumant and Wandering Activism in Cameroon
}

\author{
Ismaila Datidjo \\ Valeri Duplexe Yotedje
}

Faculté des Lettres et Sciences Humaines,

Université de Dschang, Cameroun

Armand Tchinenba

Faculté des Sciences Juridiques et Politiques,

Université de Dschang, Cameroun

\begin{abstract}
During the three (03) decades which followed Cameroon's return to plural democracy in 1990, the political arena recorded a multitude of actors and organizations with various opinions. Nowadays, more than three hundred (300) political formations have emerged with different ideologies proposed to citizens. Law $\mathrm{n}^{\circ}$ 90/053 of December 19, 1990 relating to freedom of association, supported by law $n^{\circ} 90 / 056$ of December 19,1990 relating to the creation of political parties, has promoted multipartyism and ideological development in Cameroon. Thus, it has developed behaviors of nomadism or political transhumance among many actors of the Cameroonian political scene. This article, which is a reflection on the democratic experience of Cameroonians in the context of the political multiparty system, reports on the way in which citizens militate in several formations in a cyclical manner most often or continuously. It is in these two types of behavior that transhumant and wandering activism consist respectively. They call into question the survival in the political arena of certain political forces, the weakest, that is to say those less suited to the electoral game in favor of the most powerful, therefore those who participate in political competitions and moreover, obtain
\end{abstract}


the most votes. The latter adapt by recovering the militants and sympathizers of the opposing political organizations. in an analytical approach based on the strategy of the actors of the political game involved in transhumant and wandering activism, this from field data and secondary data from the results of the elections held until then, he reports the mechanism by which certain political parties are active, while others are not, they are rather absent from competitions, one would say, they would have ceased to exist as entities.

Keywords: Transhumant militancy, wandering militancy, political parties, democracy

\section{Introduction}

L'univers politique camerounais montre à travers les comportements des sympathisants des organisations idéologiques partisanes, de multiples mutations. Celles-ci proviennent en grande partie de la reconfiguration de l'approche du jeu électoral moderne. À la suite de la seconde guerre mondiale, avec l'organisation des premières consultations électorales, l'Afrique débute une nouvelle ère de son histoire politique qui marque la « fin » du colonialisme et l'avènement d'un jeu politique progressiste. C'est ainsi qu'en Afrique francophone, la fin proclamée de la guerre froide a favorisé au début des années 1990, une nouvelle époque au cours de laquelle, les rapports aux institutions internationales dont la Banque Mondiale (BM), et le Fond Monétaire International (FMI), sont conditionnés par la mise sur pied du processus de démocratisation des États. Il s'est agi de tourner la page à la dictature militaire dans certains États et des régimes civils autoritaires dans d'autres afin d'éliminer le monolithisme encore en cours sur le continent africain. Dans cette perspective, il fallait parvenir à l'instauration des systèmes favorables à la création des partis porteurs d'opinions et d'idéologies nouvelles. (GRUENAIS \& SCHNITZER, 1995).

Dans un tel contexte de démocratisation, au Cameroun notamment, la réalité fait état de certaines pratiques en cours dans le jeu politique et qui font l'actualité dans les médias. Il s'agit précisément de la transhumance et de l'errance politiques dont les manifestations diverses procèdent de la même logique. Elles évoquent concomitamment les dissidences et les défections des militants des rangs de certaines formations idéologiques partisanes et leur adhésion dans d'autres (DIABATÉ, 2006). De même, elles rappellent les meetings d'accueil et ralliement de certains membres militants et sympathisants ; les déclarations d'alliances et fusions des partis politiques et la migration des élus pourtant investis de certains partis vers d'autres. Il faut souligner que ces faits récurrents relèvent des initiatives individuelles ou des groupes d'individus, des partis politiques et/ou des groupes de partis politiques 
dans leurs stratégies d'alliance en vue de conquérir et de dominer l'arène de compétition dans lequel ils sont engagés. (CODESRIA, 2000).

La transhumance dans son sens étymologique a une connotation agricole et concerne précisément l'activité pastorale. Dans ce sens, elle se rapporte selon (BRENAUD, 1955 : 327), à «l'ensemble des mouvements saisonniers, de rythme pendulaire et de caractère cyclique, intéressant la totalité de la masse pastorale qui l'effectue à l'intérieur des pâturages coutumiers ». Appliquée à la politique, elle se rapporte à la mobilité des personnes ou des groupes de personnes qui quittent temporairement une organisation politique pour rejoindre une autre avec pour ambition d'y satisfaire leur intérêt et de revenir au parti politique de départ. Ce que (POIRIER \& KYELEM, 2012) appellent sommairement le changement d'allégeance partisane, lequel changement d'allégeance est appréhendé comme un fléau pour la démocratie en Afrique, en ce qu'il instrumentalise les élus en quête de quelques avantages matériels et de promotion politique, affaiblit les oppositions dont les élus sont à la merci des majorités au pouvoir, fragilise les équilibres et les contrepoids nécessaires au bon fonctionnement de la démocratie et entretient l'immoralisme en politique. (BOUMAKANI, 2008).

Étant donné que la satisfaction de l'intérêt poursuivi n'est pas toujours un acquis dans les organisations politiques qui accueillent des personnes en mobilité, il va de soi qu'une situation d'insatisfaction ressentie provoque soit un retour, soit un nouveau départ. Dans le cas où l'insatisfaction entraine plutôt un nouveau départ, non pas vers le point de départ précédent, la mobilité dont il s'agit se rapporte volontiers à l'errance politique, d'où le militantisme errant. Ainsi, le militantisme transhumant désigne une mobilité de circonstance qui aboutit à un retour à son point de départ, alors que le militantisme errant, se veut être une «promenade» qui conduit son auteur d'un parti politique à un autre, sans projet de retour dans le précédent où il a milité. C'est en cela que le militantisme transhumant se distingue de celui errant, car le premier prévoit un retour, alors que le deuxième donne lieu à une série sans boucle impliquant au moins trois organisations politiques.

Dans un premier temps, ce texte présente le contexte ayant favorisé la multiplication des partis politiques et l'avènement du militantisme transhumant et errant au Cameroun ;

Dans un deuxième temps, il procède à l'analyse de ces deux modes de mobilité militante pratiqués sur la scène politique camerounaise tout en gardant à l'esprit les motivations des acteurs impliqués dans le jeu politique ;

Dans un troisième temps, il restitue les stratégies de mobilité des militants entre différentes organisations politiques et les opérations de survie 
desdites organisations affectées à la fois par la transhumance et l'errance des militants dans un contexte de compétition politique.

\section{Méthodologie}

Cet article s'appuie sur des données empiriques obtenues à partir des entretiens semi directifs tenus avec trente (30), militants et sympathisants des formations politiques dont les plus en vue représentées dans des Conseils Locaux, l'Assemblée Nationale et le Senat. Ce qui a permis d'identifier en leur sein douze personnes ayant déjà connu au moins une mobilité de type transhumant, c'est à-dire un aller-retour, et une autre de type errant, donc deux allers sans retour, ni projet de retour. L'enjeu étant de cerner les motivations qui poussent aux changements d'allégeance et surtout, les effets que lesdits changements d'allégeance ont sur la capacité d'adaptation des organisations politiques tant à l'échelle locale que nationale.

Dans le cadre de ces entretiens, des témoignages évoquant la déchéance sur le plan national et local de certaines forces politiques du fait du militantisme transhumant et errant ont été enregistrés. Aussi, il a été nécessaire d'exploiter des données secondaires issues des documents et des médias dans la perspective d'illustrer les faits tels que rapportés dans la suite du présent texte.

Cela dit, La survie des partis politiques au Cameroun confrontée à la pratique perpétuelle de la transhumance et de l'errance par les militants est la trame de cet article. Pour mener à bien la réflexion sur ce sujet, il a fallu aborder le contexte de naissance des partis politiques au Cameroun oriental et occidental pendant la période coloniale, suivre le processus qui va du multipartisme d'avant l'indépendance à celui du Cameroun moderne en transitant par le monolithisme du lendemain de l'indépendance. Ainsi, il ressort que le processus de démocratisation amorcé par le Cameroun dès 1990 a favorisé la multiplication des partis politiques et par voie de conséquence, l'avènement du militantisme transhumant et errant.

\section{Historique des partis politiques au Cameroun}

En guise de rappel, les premiers partis politiques du Cameroun français ont vu le jour en $1948^{1}$, soit une douzaine d'année avant l'accession du pays à l'indépendance. Pour l'essentiel, ces partis ont milité en faveur de la réunification avec le Cameroun britannique et pour l'indépendance.

La naissance des premières organisations à caractère politique au Cameroun britannique s'est effectuée à partir de 1939, notamment avec la C.W.U. ${ }^{2}$, soit 21 ans avant l'indépendance du pays. Au cours de son évolution,

${ }^{1}$ Union des Populations du Cameroun (U.P.C.), etc

${ }^{2}$ The Cameroons Welfare Union. 
le Cameroun britannique du sud ou southern Cameroons, correspondant de nos jours aux régions anglophones du Nord-Ouest et du Sud-Ouest, va voir naître plusieurs partis politiques cherchant à obtenir, soit son autonomie au sein de la région de l'est du Nigeria ou de la fédération nigériane, soit la séparation d'avec le Nigéria (NGOH 1996). Cet auteur souligne pour ce qui est du Northern Cameroon que, désormais rattaché au Nigéria voisin, ce n'est qu'en 1959 qu'il connait sa première organisation politique. Toutefois, l'unification du Cameroun britannique (southern Cameroons), à celui français va marquer une étape du jeu politique avec l'implantation du monolithisme. En 1960 lors de son congrès, l'U.C. ${ }^{3}$ alors circonscrite dans ce qui correspond aux régions actuelles de l'Adamaoua, du Nord et de l'Extrême-Nord, relevant du Cameroun Oriental ou français, en appelle à l'unification des forces politiques en présence. En 1962, le même appel est envoyé aux forces politiques du Cameroun britannique et finit par avoir un avis favorable ouvrant en conséquence la voie au monopartisme.

C'est en 1966, soit six années passées après l'indépendance que le premier parti unique du Cameroun unifié, l'U.N.C. ${ }^{4}$, voit le jour sous le pattern du fédéralisme. Il assemble les forces politiques à la fois du Cameroun Oriental (français), et du Cameroun Occidental (britannique), qui ont ainsi accepté leur dissolution préalable. Jusqu'en 1990, année de la réouverture de l'espace politique à la diversité des opinions, le Cameroun a évolué sous le modèle monolithique avec ses deux parties occidentale et orientale devenus un État unitaire lors du référendum du 20 mai 1972. C'est justement à partir de 1990, que le R.D.P.C. ${ }^{5}$, héritier de 1'U.N.C. en $1985^{6}$, va faire face à des rivalités, ceci du fait de la création d'organisations politiques d'opposition. Dès lors, le phénomène du militantisme transhumant et errant s'installe entre tous les partis politiques en présence sur l'arène du jeu démocratique du Cameroun moderne.

\section{Facteurs associés au militantisme transhumant et errant}

Partant du principe selon lequel le développement politique est évolutif et tend vers la plénitude de la démocratie comme finalité ultime (CHOUA BENG HUAT, 2001), les acteurs politiques camerounais assistent à la fin du monopartisme autrefois érigé en modèle de gouvernance. Cette fin du monolithisme se matérialise avec l'adoption et l'application de la loi $\mathrm{n}^{\circ}$ 090/053/ du 19 décembre 1990, marquant l'avènement du multipartisme sur le champ des compétitions électorales. Adoptée comme nouvelle formule, cette loi augure aussitôt une lueur pour l'éclosion des opinions constructives

\footnotetext{
${ }^{3}$ L'Union Camerounaise

${ }^{4}$ L'Union Nationale Camerounaise

${ }^{5}$ Le Rassemblement Démocratique du Peuple Camerounais

${ }^{6}$ Lors du congrès dit du renouveau de 1985 , tenu à Bamenda
} 
dans le jeu politique et le processus du développement inclusif du Cameroun. Dans sa phase d'implémentation, elle favorise la création de plusieurs organisations porteuses d'idéologies politiques. Dès lors, le paysage politique est reconfiguré et donne lieu entre 1990 et 2010, à la naissance de plus de 300 formations politiques, avec 271 légalisés selon l'INS ${ }^{7}$ (2011). Cette démocratisation selon (VAN de WALLE \& SMIDDY, $2000: 41$ ), a «balayé les vieux régimes à parti unique et les a remplacés par des systèmes politiques multipartites plus concurrentiels(...)».

Malgré le retour au multipartisme du Cameroun moderne, toutes les forces politiques légalisées n'ont pas réussi à s'imposer sur l'espace des jeux électoraux en l'occurrence. Pendant les élections, il s'observe une sousreprésentativité des partis politiques aux différentes compétitions. Aussi, au regard du nombre de partis politiques légalisés, une très faible représentativité à l'Assemblée Nationale est visible ${ }^{8}$, et donc la non-implication du plus grand nombre dans la gestion des affaires de la nation. À titre indicatif, lors des présidentielles de 1992, 1997, 2011 et 2018, respectivement, $06^{9}, 07^{10}, 23^{11}$, et $09^{12}$ organisations politiques ont participé à ces compétitions. De même, les législatives de 1992, 1997, 2002, 2007, 2012 et 2020 ont dans l'ordre enregistré les chiffres suivants de partis politiques représentés à l'Assemblée Nationale : $06^{13}, 10^{14}, 05^{15}, 05^{16}, 07^{17}$ et $08^{18}$. Il en découle de ces observations que depuis le rétablissement de la démocratie plurielle au Cameroun, seules 37 formations politiques ont participé aux présidentielles et 15 aux législatives, de très faibles taux au regard du nombre de partis politiques légalisés. Ce qui témoigne de l'absence du plus grand nombre, «les inadaptés », aux différentes échéances électorales organisées depuis lors.

Cette absence notoire du jeu électoral de nombre d'organisations politiques, s'illustre d'emblée comme incitatrice à la transhumance et/ou à l'errance de leurs militants. Si la procédure de participation reste ardue, voire

\footnotetext{
${ }^{7}$ Se dit de l'Institut National de la Statistique du Cameroun.

${ }^{8}$ Les données qui suivent sont disponibles entre autres dans archive des résultats des élections parlementaires au Cameroun sur :

https://www.archive.ipu.org/parline-f/reports/1053 arc.htm

${ }^{9}$ R.D.P.C., S.D.F., U.N.D.P., U.D.C., M.P. et R.F.P.

${ }^{10}$ R.D.P.C., U.P.C., M.D.R., P.P.D., A.M.E.C, R.D.P.F.et D.I.C.

${ }^{11}$ R.D.P.C., S.D.F., A.D.D.,U.D.C., P.A.P., C.P.P., LA DYNAMIQUE, P.A.D.D.E.C, M.P., A.F.P., B.R.I.E.C., U.F.P., M.A.N.I.D.E.M., U.F.D.C., M.E.C., F.U.C., M.E.R.C.I., U.P.A., G.C., C.N.C., S.L.C., A.M.E.C.et P.S.U.

${ }^{12}$ R.D.P.C., M.R.C., UNIVERS, S.D.F., U.D.C., A.D.D., M.C.N.C., PURS et NOW!

${ }^{13}$ R.D.P.C, U.N.D.P., U.P.C., M.D.R., P.D.C. et P.N.P.

${ }^{14}$ R.D.P.C. S.D.F., U.N.D.P., U.P.C., U.D.C., M.D.R., A.F.D.P., U.F.D.C., L.M.C.Y., P.D.C.

${ }^{15}$ R.D.P.C., S.D.F., U.D.C., U.P.C., U.N.D.P.

${ }^{16}$ R.D.P.C., S.D.F., U.N.D.P., U.D.C. et M.P.

${ }^{17}$ R.D.P.C., S.D.F., U.N.D.P., U.D.C., U.P.C., M.R.C. et M.D.R.

${ }^{18}$ R.D.P.C., U.N.D.P., S.D.F., P.C.R.N., U.D.C., F.S.N.C., M.D.R. et U.M.S.
} 
impossible parce que la valeur de la caution à déposer pour le dossier de candidature paraît élevée à en croire certains leaders et membres de partis politiques rencontrés ${ }^{19}$, il faut reconnaitre qu'ils avouent par-là leur incapacité à se trouver sur l'arène de compétition par faute de moyens financiers nécessaires. Ceci induit aussitôt leur inadaptation aux règles du jeu en place. Généralement ces partis de faible ancrage social sont subséquemment d'une faible représentativité sur le plan national.

De ce qui précède donc, il y a lieu de le souligner que l'inadaptation des formations politiques, notamment celles de l'opposition aux règlements des compétitions électorales qu'elles ne se privent pas très souvent de critiquer, implique inéluctablement leur non-implication effective à la vie politique républicaine de la Nation. Cette non-implication se confirme d'ailleurs dès lors que ces partis optent pour la politique de la chaise vide en appelant au boycott ${ }^{20} \mathrm{du}$ jeu électoral organisé. En choisissant le boycott, les formations politiques, hypothèquent à coup sûr la chance des militants désireux de se réaliser du point de vue politique. Ce qui constitue une source de désappointement pour certains militants qui se donnent le choix de la désertion et donc de la transhumance et/ou de l'errance. Plus un tel choix est important au sein des membres militants d'une formation politique, plus celleci perd en potentialité et cours inévitablement vers son effacement. Le boycott des élections de 1992 par seize (16) partis politiques dont le S.D.F. alors principale force politique de l'opposition en est une illustration, car selon certains anciens et actuels sympathisants du S.D.F., ce parti a perdu de très nombreux militants au profit d'autres partis politiques dont le R.D.P.C. au pouvoir. Tandis que le M.R.C. paraissait à sa création en 2012 comme point de chute de nombreux militants déçus du S.D.F., lui aussi, pour avoir provoqué des déceptions dans son rang en boycottant les législatives et municipales de février 2020, s'est mis aussitôt à enregistrer des désistements et démissions des militants et sympathisants de ses rangs, ceci au profit d'autres partis à l'instar du P.C.R.N., etc.

Pour les Camerounais attachés à la chose politique et pour ceux ayant des ambitions de visibilité politique, ou de participation à la vie des communautés nationales à l'échelle locale ou supra locale, il devient inconcevable de militer au sein des partis qui n'existent pas ou qui n'existent que du point de vue théorique sans véritable ancrage dans les sphères de prise

\footnotetext{
${ }^{19}$ Ils estiment que les cautions exigées sont fixées pour dissuader certains candidats et partis politiques. Comme indication, lors de la présidentielle de 2018, le montant requis s'élevait à 30000000 de francs CFA.

${ }^{20}$ Un cas illustratif est celui du Social Democratic Front (S.D.F.), lors des élections couplées de 1992 et récemment, le cas du Mouvement pour la Renaissance du Cameroun (M.R.C.), qui a boycotté les législatives et municipales couplées de févier 2020, y compris les régionales en vue de décembre 2020.
} 
de décisions. D'ailleurs, faut-il le dire, un parti politique vivant c'est un parti ambitieux qui s'emploie au-delà de toutes les péripéties, à exister, à vivre, donc à être actif. À s'en tenir aux données de terrain et selon les principes de la démocratie, la matérialisation des ambitions d'un parti politique passe sans doute par sa participation aux différentes compétitions politiques, seules trajectoires pour conquérir le pouvoir, gagner en notoriété et susciter l'adhésion de nouveaux militants. Au cas contraire, le départ des militants acquis vers des forces politiques plus attrayantes devient conséquemment inévitable.

\section{Militantisme transhumant et errant en périodes électorales : causes et manifestations}

Le terme transhumance du point de vue étymologique relève du domaine agricole et renvoie au comportement des peuples pasteurs partant d'une contrée à une autre au rythme des saisons, en quête de verdoyants pâturages pour leurs troupeaux. Ils ont beau faire de longs voyages avec leurs bêtes, ils reviennent toujours au " pays ", même s'il faut reprendre plus tard leurs pérégrinations, peut-être dans d'autres directions pour la même cause. (TIENSIGUE DIABATÉ, 2006). Appliquée à la politique, l'expression de transhumance révèle un autre comportement qui lui est très proche. Il s'agit de l'errance politique ; celle-ci se traduit aussi par le déplacement des personnes à la quête des avantages liés à l'adhésion et au militantisme au sein des organisations d'opinions. Pourtant, à la différence de la transhumance, l'errance politique ne donne pas lieu au retour dans son parti de départ. Elle fait état de déplacements continus de parti politique en parti politique, sans intention de retour à la case de départ. Toujours est-il que ces deux phénomènes s'accompagnent et se tiennent sur la base des facteurs à l'origine de leur mise sur pied par les membres militants et sympathisants des forces politiques rivalisant au nom de la démocratie plurielle. Le constat d'ensemble qui se dégage montre que ces deux formes de mobilité politique ne sont pas l'exclusivité des catégories spécifiques. Les hommes et les femmes, au même titre que les jeunes soucieux de leur propre réalisation en tant qu'opérateurs politiques en sont concernés. Tous ne se privent point de changer d'allégeance politique dès lors que l'occasion se présente à eux. Ainsi, faut-il le souligner, les mobilités militantes s'enregistrent aussi bien entre les partis de l'opposition et le parti politique au pouvoir dans les deux sens, qu'entre ceux de l'opposition entre eux.

\subsection{Causes de la transhumance et de l'errance politique}

Comme le démontrait déjà (TIENSIGUE DIABATE, 2006), les causes de la mobilité des militants d'opinions entre les forces politiques peuvent être recherchées dans l'histoire. À cet effet, il s'en suit que l'observation du 
phénomène au Cameroun fait état de plusieurs réalités qui se tiennent les unes les autres. Le paragraphe suivant tente de revisiter cet aspect. De l'avis de personnes interrogées dans le cadre de cette réflexion, l'absence d'une véritable idéologie, autrement appelée "projet de société convainquant ${ }^{21}$ porté par les partis politiques et leurs membres, favorise le départ des militants d'un parti politique pour un autre. Un projet de société inexistant, méconnu ou mal connu des militants, ne participe pas à la rétention de ces derniers, surtout pas ceux ayant le discernement et dont les actions voudraient reposer sur des convictions idéologiques claires. (ISMAÏLA DATIDJO \& YOTEDJE, 2020). L'absence ou l'inadéquation de la contrepartie par rapport aux efforts consentis et aux attentes et aspirations des militants entretient en eux le désir de désertion pour rejoindre les rangs d'autres forces politiques.

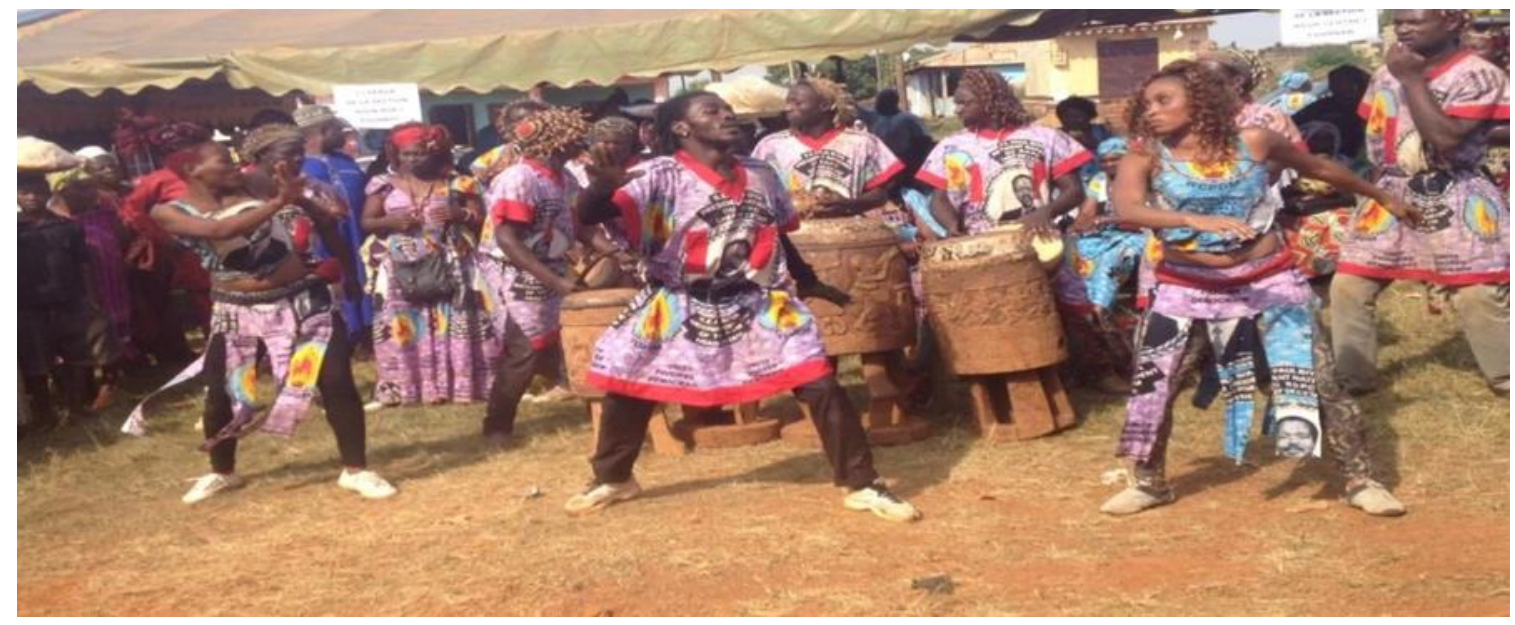

Photo : cérémonie d'accueil de nouveaux militants RDPC en provenance de l'UDC.

Source : (MENGOUEN NCHOUWAT KENSOUMI, 2017 : 34)

Ces militants, le plus souvent en quête de positionnement sur l'échiquier politique local ou national pour les uns, à la recherche d'une visibilité de prestige pour les autres etc, ne manquent pas un tant soit peu, de traduire leur déception par la désertion d'une organisation politique en faveur d'une autre. C'est la substance de l'image ci-dessus présentée où plusieurs nouveaux militants du RDPC en provenance de l'UDC, sont accueillis.

Certains militants avides de pouvoirs et parfois animés par "l'impatience du désir », ne supportent pas d'être toujours du côté des perdants lors des échéances électorales ; ils préfèrent quitter ces partis, disentils «inadaptés à la concurrence », pour rejoindre les rangs des partis de renommée pour ainsi espérer y assouvir leurs désirs et satisfaire leurs attentes.

${ }^{21}$ Il s'agit d'un programme politique qui cadre avec les aspirations du peuple dans son ensemble. 


\subsection{Manifestations du militantisme transhumant et errant en contexte électoral}

Bien que la transhumance résulte de causes diverses telles que rapportées plus haut, il n'en demeure pas moins que certains acteurs, précisément « les militants de second rang », c'est-à-dire ceux n'ayant pas de positions privilégiées au sein de leurs formations politiques, désertent ponctuellement lesdites formations avec pour ambition d'obtenir des avantages personnels en militant en faveur d'autres forces en compétition. À ce titre, il y a lieu de souligner que les campagnes électorales riment toujours avec des dons divers offerts par les partis politiques aux militants qui se déploient sur le terrain dans l'optique de persuader l'électorat. (ISMAÏLA DATIDJO \& YOTEDJE, 2020).

Depuis le retour du Cameroun au multipartisme, des faits récurrents de l'actualité politique nationale qui, malgré leur constante répétitivité sont restés insignifiants, ce sont les phénomènes de militantisme transhumant et errant des membres et sympathisants des forces politiques concurrentes. La logique qui sous-tend la mobilité des acteurs et groupes d'acteurs politiques qui s'y consacrent, réside dans le fait que ces derniers quittent temporairement ou définitivement une organisation politique pour rejoindre une autre avec l'intention d'y satisfaire leurs intérêts. Il s'agit de faire valoir leurs aspirations personnelles et de bénéficier au maximum des opportunités éventuelles qui s'offriraient à eux. Ce qui est devenu une pratique rependue dans un contexte particulier où un militant de gagné ou de convaincu en vaut plusieurs, car il peut aussi mener avec lui d'autres militants et augmenter par-là le potentiel de votants pour un parti politique déterminé.

Les phénomènes de la transhumance et de l'errance des militants de partis politiques au Cameroun puisent dans des situations favorables telles que les scissions des partis politiques, elles-mêmes résultantes des querelles intestines qui y ont cours. Ces phénomènes découlent également du sentiment d'insatisfaction ressenti par les membres et militants ambitieux et soucieux de se réaliser en tant qu'opérateurs politiques, mais qui n'y trouvent pas de tremplin au point de se sentir obligés de quitter pour tenter leur chance ailleurs. Toujours est-il que leur départ du parti ainsi délaissé est volontaire, tant aucune force contraignante ne s'impose dans ce sens à eux. Aussi, il faut y voir l'insatisfaction ressenti par les militants et sympathisants admis comme de «second rang» qui désertent de leurs forces politiques d'adhésion pour rejoindre de nouvelles qui leur accorderaient plus de considération et accompagneraient leurs efforts à travers des ténors lors des échéances électorales où ils se singularisent par un militantisme agissant. Ainsi, les militants très en vue qui décident de quitter un parti politique, le font par démission officielle ou informelle, mais très souvent rapportée par les médias locaux ou nationaux. Par contre, les autres militants, donc ceux de «second 
rang », procèdent par abandon, et leur départ peut ne pas être constaté. L'éventuel constat résulterait seulement d'un départ de masse qui se fait remarquer au cours des opérations de séduction où ils sont généralement sollicités. S'il est vrai que les personnages les plus en vue dans les partis politiques les quittent en démissionnant, il ne faut pas perdre de vue qu'il arrive plutôt qu'ils soient déchus ou révoqués par l'instance juridique du parti politique suite à un comportement non orthodoxe. Toutefois, quel que soit le motif de départ d'un militant d'une force politique pour une autre, qu'il s'agisse d'un ténor ou d'un membre militant ou sympathisant de «second rang ", il devient désormais libre de rejoindre un autre parti politique ou d'en créer soi-même son propre parti.

De manière générale, on observe au regard du jeu politique au Cameroun, deux types de mobilité des militants à savoir celui volontaire à motivation personnelle et celui non volontaire, c'est-à-dire forcé.

- Dans le premier type, le départ se produit à la suite d'un sentiment de délaissement qu'éprouve parfois le militant vis-à-vis du mode de fonctionnement du parti qui, de l'avis des informateurs, ne leur prête pas l'attention qu'ils méritent. De ce fait, l'acteur politique concerné quitte son parti d'origine pour un autre de son choix sans aucune contrainte extérieure.

- Le second type quant à lui, quoique rarement observé dans le paysage politique camerounais, reste réel dans le jeu démocratique actuel. Il n'intervient qu'en situation de dérive de comportements dérogeant à l'éthique ou à la déontologie du parti par un membre militant ou cadre ou sympathisant dudit parti politique. Par la suite, en cas de nonrespect de la discipline du parti, le contrevenant est frappé de sanctions dont l'une des plus sévères est son expulsion. À titre d'exemple, le rapport de la commission de discipline du comité central du R.D.P.C. ayant statué sur les cas d'indisciplines relevées lors du processus de déroulement des élections municipales du 09 février 2020 et rendu public par son Secrétaire Général, fait état de 78 militants et militantes qui ont été sanctionnés. Ces sanctions sont entre autres constituées des cas de rappel à l'ordre, des déchéances de fonctions, des exclusions temporaires d'une durée allant de $06,12,24$ à 36 mois, et des exclusions définitives ${ }^{22}$. À travers cette décision, les ténors dudit parti en rappellent non seulement à la responsabilité de leurs camarades militants, mais aussi et surtout, ils réaffirment la place prépondérante de la discipline dans les activités politiques. Ce type d'acte est généralement à l'origine des départs ou migrations forcées de militants

${ }^{22}$ Quotidien Cameroon-tribune du 10 septembre 2020 consultable en ligne, au : https://www.cameroon-tribune.cm/article.html/34834/fr.html/rdpc-78-militants 
ainsi incriminés pour d'autres formations politiques sans perspective de retour possible. En cas d'expulsion, deux cas de figures se dessinent. Soit le militant quitte le parti dans lequel il lui est reproché des écarts de comportements pour rejoindre un autre qui lui offre plus de visibilité, plus d'opportunité et donc un surcroit de considération, soit il quitte ce parti pour créer son propre parti politique s'il en a les moyens nécessaires.

Un autre cas de mobilité à caractère volontaire est celui de tout militant qui, suite à sa propre appréciation de la réalité vécue au sein de son parti politique d'adhésion, ceci du fait que sa conviction idéologique ne peut y être considérée et instituée, décide de son plein gré de s'en aller pour rejoindre le rang d'une autre organisation à idéologie apparentée ou similaire à la sienne. Les exemples pour ce cas sont courants. Il convient d'évoquer ici le cas de l'ancien conseiller municipal du S.D.F., à la mairie de Douala I ${ }^{\text {er, Célestin }}$ DJAMEN qui, après sa démission de son parti d'origine, a rejoint les rangs du MRC où, avant sa nouvelle démission, exerçait au sein de son directoire au poste de Secrétaire National en charge des Droits de l'homme et de la Gouvernance. Il en est de même de l'annonce de sa démission par Jean Jacques EKINDI, ex coordonnateur et fondateur du $\mathrm{MP}^{23}$, faisant ainsi son retour au sein du R.D.P.C. ${ }^{24}$, un parti qu'il avait pourtant quitté pour fonder le sien. Tel est également le cas du P.C.R.N. dont le président, Cabral LIBII a quitté le parti UNIVERS qui l'a d'ailleurs porté candidat lors des investitures pour la présidentielle d'octobre 2018.

À titre de rappel, dans le cas où une situation vécue entraine la radiation ou le départ volontaire d'un militant, et que ce dernier effectue un autre départ, non pas dans le sens du retour au sein de la précédente formation politique où il a milité, mais dans celui de la continuité pour rejoindre une énième force porteuse d'opinion politique, la mobilité dont il s'agit se rapporte volontiers à l'errance politique. Celle-ci peut donc être la résultante d'une suite de déceptions qui entraine par voie de conséquence des départs consécutifs de parti politique en parti politique.

\section{Stratégies de survie des partis politiques face au militantisme transhumant et errant}

La non-participation de certains partis politiques aux élections conduit inéluctablement à leur mise à l'écart en ce qui concerne la gestion des affaires publiques. Ainsi, afin d'assurer leur existence sur la scène politique nationale

\footnotetext{
${ }^{23}$ Mouvement Progressiste.

${ }^{24}$ ESSOGO Armand écrit: «Jean Jacques EKINDI rentre au R.D.P.C.». Quotidien Cameroon-Tribune du 04 mai 2020. Consultable sur https://www.cameroontribune.cm/article;html/32167/fr.html/jean-jacques-ekindi-rentre-au-rdpc.
} 
malgré leur auto-exclusion par le recours à la tactique de la chaise vide, ceuxci mettent communément en œuvre des stratégies dont la plus en vue porte sur les jeux d'alliance ou de coalition entre partis de l'opposition d'une part ou entre quelques-uns de ces derniers et le parti au pouvoir d'autre part.

À défaut de pouvoir conquérir de nouveaux espaces en enregistrant des scores sur des terrains tenus par les forces politiques adverses, une autre stratégie de survie des partis en perte de notoriété dans l'arène des compétitions électorales consiste en la conservation des fiefs dits électoraux qui sont pour eux des acquis, des socles sur lesquels au moins, ils expriment leur existence en tant organisations politiques. Pour illustration, la région du Nord-Ouest est une «aire politique attribuée au S.D.F.» (ZAMBO BELINGA J. M. 2007 : 12).

Au même titre que le Social Democratic Front (S.D.F.), dans les zones anglophones, les autres partis politiques suivants ont presque toujours comptabilisé leurs meilleurs scores au terme des compétitions politiques dans leurs fiefs ou pré-carrés électoraux traditionnellement acquis. L'Union Démocratique du Cameroun (U.D.C.), perçue comme le parti du peuple bamoun dans son terroir le Département du Noun; le Rassemblement Démocratique du Peuple Camerounais (R.D.P.C.), qualifié globalement de parti «béti », dans les régions du Centre et du Sud; l'Union pour la Démocratie et le Progrès (U.N.D.P.), parti dit des Foulbé ou des nordistes dans les zones septentrionales; l'Union des Populations du Cameroun (U.P.C.), considérée comme le parti des Bassa, en pays bassa ; le Mouvement pour la Défense de la République (M.D.R.), compris comme le parti des communautés «kirdi », dans les localités du septentrion à dominance kirdi; etc. (MENTHONG, 1990). En plus d'enregistrer leurs meilleurs scores, ces formations politiques ont presque toujours remporté les élections dans leurs contrées respectives lors des échéances électorales.

\subsection{Coalitions comme stratégies de conquêtes politiques et de survie des partis}

Au cours des opérations électorales, des alliances de partis politiques s'observent sur la scène nationale. Dans la plupart de cas, elles sont établies sur la base des intérêts et aspirations réciproques des contractants qui entendent par-là exister en se soutenant mutuellement. Par ce procédé, les partis coalisés démontrent qu'en allant en compétition étant esseulés, les chances de convaincre l'électorat et d'obtenir de bons scores sont limitées. Il s'agit donc de se renforcer réciproquement pour faire face soit à d'autres jeux d'alliances adverses ou à des partis plus imposants. Une analyse de la réalité dénote deux types d'alliances ou de coalitions, celles mettant en jeu les partis de l'opposition entre eux et celles rapprochant certaines formations politiques de l'opposition et le parti au pouvoir. De ces deux formes d'alliances, il se 
dégage que les accointances d'idéologies ne sont pas des facteurs mis en avant, mais plutôt les espoirs des uns et des autres.

\subsubsection{Coalitions des partis de l'opposition}

Ostensiblement, les alliances des partis politiques de l'opposition au Cameroun se sont toujours constituées pour opposer une force à la mesure de celle du parti au pouvoir qui compte son électorat particulièrement sur toute l'étendue du territoire national. Lors de la présidentielle de 2018, des partis de l'opposition se sont constitués en coalition pour soutenir la candidature du président du M.R.C. Certes l'alliance M.R.C. ${ }^{25}-N O W^{26}$-M.P.C.N. ${ }^{27}$ etc, ambitionnait de renverser un régime qui selon les militants de ces partis, durait déjà trop. Il ressort que ce jeu d'alliance est révélateur d'un autre fait. Les partis de petite visibilité entendent par-là assurer leur survie en cheminant avec ceux étant les plus en vue. De l'avis des militants de partis rencontrés, alors que certains partis aspirent à conquérir le pouvoir, d'autres cherchent plutôt à assurer leur survie politique compte tenu de leur potentiel électoral réduit qu'ils acceptent de fusionner avec ceux des partis alliés.

Les alliances de survie sont généralement constituées par des partis de faible visibilité qui ne disposent pas de grands électorats ou dont l'électorat est principalement circonscrit dans leurs fiefs dits électoraux et qui entendent profiter des plus forts pour se faire une notoriété accrue auprès des citoyens. Le cas du M.P.C.N. dont le président Paul Éric KINGUE a été admis directeur de campagne du M.R.C. en tant que tête de la coalition à la présidentielle d'octobre 2018 en est un exemple. Alors que lui-même préside une formation politique qui n'a pu briguer la magistrature suprême, il milite par alliance en faveur du candidat Maurice KAMTO du M.R.C. En effet, ce parti va perdre l'élection face au R.D.P.C. avec 71, $28 \%$ de suffrage contre 14,23\%. L'alliance n'étant que circonstancielle, le président du M.P.C.N va se lancer sous la bannière de son propre parti politique désormais adapté à la conquête de la mairie de Djombé-Penja son fief électoral, ceci malgré l'appel au boycott lancé par son parti d'alliance à la présidentielle. Il sera, après de multiples «propagandes d'humiliations et de sabotages $»^{28}$ de l'image du président de

\footnotetext{
${ }^{25}$ Mouvement pour la Renaissance du Cameroun.

${ }^{26}$ Mouvement politique dont Akere MUNA est leader. Ce mouvement est une organisation dite de citoyens engagés qui compte dans ses rangs plusieurs fondateurs et leaders d'autres forces politiques, notamment l'Union des Populations du Cameroun (U.P.C.), et l'Alliance des Forces Progressistes (A.F.P.), accompagnés de plusieurs autres petites structures.

${ }^{27}$ Mouvement Patriotique pour un Cameroun Nouveau.

${ }^{28}$ En effet, les leaders du MPCN, du MRC et autres acteurs clés des formations politiques de l'opposition ont, après avoir essuyé la défaite lors de l'élection présidentielle d'octobre 2018, lancé un vaste mouvement de revendication baptisé «non au hold-up électoral » auquel les populations à l'échelle nationale et internationale ont adhéré. Ceci va leur valoir après arrestation, 9 mois de prison pour certains, et 12 mois pour d'autres. Jusqu'à présent (2021)
} 
son ex-parti de la coalition, élu à la fonction de maire de la commune pour laquelle il s'est présenté en tant que candidat.

Les jeux de coalitions naissent généralement de l'incapacité reconnue des acteurs à réaliser de façon solitaire leurs souhaits de conquête et/ou de maintien au pouvoir eu égard les limites dans leur capacité de recueillir les voix nécessaires lors des opérations de votes. Selon (SINDJOUN, 1997 : 91), "La compétition électorale apparait comme une procédure de conquête et légitimation du pouvoir ». Conscients de fait, les partis politiques faibles et désireux de contribuer à la vie politique de la nation se trouvent dans l'obligation de nouer des alliances. Celles-ci se mettent en place, que ce soit entre partis de l'opposition dans un cas, ou entre certains partis de l'opposition et le parti politique au pouvoir dans un autre cas, dans l'optique d'opérer des actions stratégiques communes.

\subsubsection{Alliances entre les partis de l'opposition et le parti au pouvoir}

La coalition de certains partis de l'opposition avec le parti au pouvoir le RDPC, n'est pas un phénomène nouveau. Pour des raisons diverses selon les acteurs, des formations politiques de l'opposition rejoignent les rangs du R.D.P.C. Pour de nombreux informateurs, cette alliance se justifie par le pacte de non trahison que les partis signent ${ }^{29}$ avec le R.D.P.C. d'une part, et également par la lutte de conquête des postes et des positions de pouvoir, la quête de l'intérêt personnel ou de groupe d'autre part. Il s'agit de retenir qu'en plus de la visibilité et de la notoriété recherchée par les partis alliés du R.D.P.C. au Pouvoir, les ténors desdits partis de l'opposition entendent obtenir des positions de pouvoir au sein du système administratif en guise de récompense pour le ralliement de leurs organisations politiques. En exemple, suite à leur alliance avec le R.D.P.C. au pouvoir lors de la présidentielle de 2018, Jean de Dieu MOMO de la PADDEC ${ }^{30}$, Issa TCHIROMA BAKARY du F.S.N.C ${ }^{31}$ et Bello Bouba MAÏGARI de 1'U.N.D.P ${ }^{32}$ entre autres, font partie du gouvernement ainsi constitué.

La coalition de 2018 lors de la présidentielle ${ }^{33}$ n'est pas la première pour le parti au pouvoir au cours de son histoire. Bien avant, plusieurs

beaucoup croupissent encore dans les geôles des prisons. Ce que beaucoup de leaders de ladite coalisions à l'instar de président du MPCN n'ont pas pu digérer au regard de ses multiples sorties médiatiques de décryptage des faits y relatifs.

${ }^{29}$ MAMAN (2008), « les alliés du R.D.P.C. en bonne place », in Cameroun tribune, publié [en ligne] sur https://www.cameroun-tribune.cm/article.html/21002/fr.html/alliés-du-rdpc-enbonne-place, le 01 Octobre 2018 à 16 h15 et consulté le 09 fév. 2018.

${ }^{30}$ Le parti des Patriotes Démocrates pour le Développement du Cameroun.

${ }^{31}$ Le Front pour le Salut National du Cameroun.

${ }^{32}$ L'Union Nationale pour la Démocratie et le Progrès.

${ }^{33}$ Une coalition de 20 partis politiques s'est constituée pour soutenir le candidat du R.D.P.C. et ainsi faciliter sa victoire. 
mouvements politiques ont inféodés ses rangs ${ }^{34}$ au point de paraitre aux yeux de nombre de Camerounais plutôt comme des démembrements du R.D.P.C. et non comme des forces d'opinions qui lui sont opposées. Par leur allégeance exprimée au R.D.P.C., ces partis sont pour la plupart actifs et d'ailleurs impliqués dans la gestion des affaires publiques et politiques du fait de leur présence au sein des chambres parlementaires, c'est-à-dire à l'Assemblée Nationale et au Sénat, y compris dans les CTD ${ }^{35}$.

D'un côté comme de l'autre des formes de coalitions sus évoquées, les partis politiques incluant celui au pouvoir et ceux de l'opposition se composent en forces dont les ambitions politiques restent avérées. Conquérir le pouvoir et le tenir le plus longtemps possible pour les forts ou assurer leur survie et se procurer une notoriété de surcroit pour les moins forts. À côté, il faut garder à l'esprit les intérêts individuels que poursuivent çà et là les acteurs politiques qui se meuvent dans l'arène du jeu politique et qui ne se privent pas parfois de changer de parti politique au gré des intérêts personnels qu'ils poursuivent dès lors que l'opportunité se présente à eux.

D'une part, les jeux d'alliance sont devenus une expression de la volonté de survie pour les partis politiques les moins en vue qui tiennent à exister politiquement malgré leur manque ou insuffisance de ressources ; d'autre part, les coalitions permettent aux partis politiques les plus en vue de capitaliser l'électorat nécessaire en perspective de la conquête du pouvoir et de leur maintien en des positions privilégiées sur l'échiquier politique national.

\subsection{Survie des partis politiques par la protection des fiefs électoraux}

Plusieurs partis possèdent dans le champ politique actuel, ce qu'il convient d'appeler à l'observation empirique des faits, un fief électoral. Il s'agit en effet d'une aire ou d'un environnement politique acquis soit par l'adresse dans le jeu politique, soit par le simple fait qu'il serait le terroir de provenance du fondateur ou du président du parti, le quel parti selon la réalité au Cameroun tend généralement à se confondre en priorité à une organisation à fondement ethno-tribal et régional (ISMAÏLA DATIDJO \& YOTEDJE 2020). Ces fiefs sont entretenus avant, pendant et après les périodes de compétitions électorales par des hommes politiques aux moyens de plusieurs stratagèmes pas toujours règlementaires (TSANA NGUEGANG 2015). Généralement qualifiées de base-arrières des partis politiques, certaines circonscriptions ont été investies par des leaders, élites ou personnalités influentes de la contrée. En tant que membres, militants et/ou sympathisants des formations politiques, et compte tenu de leur influence et de la sympathie

\footnotetext{
${ }^{34}$ Il s'agit entre autres de l'U.P.C., A.N.D.P., l'U.N.D.P., le M.D.R. et le F.S.N.C., etc.

${ }^{35}$ Se dit des Collectivités Territoriales Décentralisées.
} 
que les populations développent à leur égard, ils assurent le maintien de la présence de leurs forces politiques en ces milieux.

Les partis politiques au Cameroun, qu'ils soient au pouvoir ou dans l'opposition, possèdent donc, au regard de la filiation d'appartenance de leurs membres et/ou de leurs sympathisants, à des étendues très souvent identifiables aux contrées de provenance des communautés ethniques et culturelles desdits membres et sympathisants où ils exercent leur influencent dans le sens de les conserver comme leurs chasse-gardées. Ils n'entendent pas y perdre de leur prépondérance et s'emploient à la monopolisation de ces milieux qui se rapportent à des circonscriptions électorales qu'ils assimilent aux fiefs de leurs partis politiques.

De ce qui précède, il se révèle que des partis politiques se battent pour remporter les élections dans leurs fiefs. Devant cet état de fait, force est de constater au regard des présidentielles, législatives et/ou municipales, que la dynamique des formations politiques les pousse à se mobiliser pour remporter au moins les élections dans leurs fiefs respectifs. À titre indicatif, il convient de citer entre autres le S.D.F. dans les régions du Nord-Ouest et Sud-Ouest, le $\mathrm{MRC}$ dans la région de l'Ouest excluant le Département du Noun presque totalement acquis à l'U.D.C. le P.C.R.N. dans la circonscription Wouri-est etc. À défaut de pouvoir gagner les élections dans leurs fiefs respectifs, ces partis politiques engrangent leurs plus grands scores dans leurs base-arrières à partir desquelles ils entretiennent toujours l'espoir de rebondir pour conquérir éventuellement d'autres contrées. 
Scores des partis politiques par régions à la présidentielle d'octobre 2018

Source : Agence Cameroun Presse ${ }^{36}$

\begin{tabular}{|c|c|c|c|c|c|c|c|c|c|c|}
\hline & Scores par 1 & gions en & urcent & es (\%) & & & & & & \\
\hline $\begin{array}{l}\text { Partis } \\
\text { politiques }\end{array}$ & Adamaoua & Centre & Est & $\begin{array}{l}\text { Extrême } \\
\text { Nord }\end{array}$ & Littoral & Nord & $\begin{array}{l}\text { Nord- } \\
\text { Ouest }\end{array}$ & $\begin{array}{l}\text { Sud- } \\
\text { Ouest }\end{array}$ & Ouest & Sud \\
\hline M.R.C. ${ }^{37}$ & 2,61 & 15,25 & 2,63 & 3,45 & 38,6 & 4,15 & 3,6 & 3,54 & 30,56 & 2,15 \\
\hline UNIVERS & 11,33 & 9,66 & 3,77 & 2,81 & 12,79 & 5,77 & 1,18 & 1,71 & 1,44 & 2,75 \\
\hline S.D.F. ${ }^{38}$ & 1,88 & 2,11 & 0,91 & 0,98 & 9,06 & 2,03 & 10,4 & 12,72 & 5,2 & 0,99 \\
\hline M.C.N.C. ${ }^{39}$ & 0,56 & $\mathbf{0 , 3 8}$ & $\mathbf{0 , 3 3}$ & 0,48 & 1,18 & 1,06 & 1,15 & 1,65 & 0,76 & 0,28 \\
\hline U.D.C. ${ }^{40}$ & $\mathbf{0 , 2 7}$ & $\mathbf{0 , 3 1}$ & 0,24 & $\mathbf{0 , 3 5}$ & $\mathbf{0 , 7 6}$ & $\mathbf{0 , 5 2}$ & $\mathbf{0 , 3 9}$ & 0,65 & 10,4 & $\mathbf{0 , 5 3}$ \\
\hline R.D.P.C. ${ }^{41}$ & 79,77 & 71,1 & 90,43 & 89,21 & 35,75 & 81,62 & 81,7 & 77,69 & 48,19 & 92,91 \\
\hline A.D.D. ${ }^{42}$ & 2,6 & 0,67 & 1,14 & 1,77 & 0,85 & 2,92 & 0,41 & 0,63 & 2,5 & 0,21 \\
\hline PURS $^{43}$ & 0,56 & 0,39 & 0,41 & 0,62 & 0,67 & 1,1 & 0,28 & 0,57 & 0,48 & 0,13 \\
\hline$N O W^{44}$ & 0,21 & $\mathbf{0 , 1 3}$ & 0,14 & 0,32 & 0,34 & 0,52 & 0,83 & 0,84 & $\mathbf{0 , 5 3}$ & 0,04 \\
\hline
\end{tabular}

Ce tableau conçu à partir des résultats de l'élection présidentielle d'octobre 2018 au Cameroun, montre qu'à des exceptions près, chaque parti politique possède un fief électoral où il réalise ses meilleurs scores. À défaut d'y avoir des élus lors d'autres opérations de votes, il se fait une estimation de son potentiel électoral qui lui garantit l'espoir d'exister en tant qu'organisation porteuse d'opinion au sein de l'arène politique nationale de plus en plus morcelée. Le fief d'un parti politique se circonscrit généralement à la région à connotation ethnico-culturelle à laquelle appartient son leader. Il peut aussi se rapporter à un milieu où les ressortissants du même terroir que ledit leader sont considérablement implantés, et parfois, à une circonscription électorale où le parti bénéficie de la sympathie des populations. Le parti R.D.P.C. au pouvoir, malgré sa présence avérée sur toute l'étendue du Cameroun en tant que «parti d'État », ne déroge pas véritablement à cette réalité étant donné les scores qu'il enregistre dans les régions du sud du pays où il affirme son enracinement ethno-tribal (ISMAÏLA DATIDJO et YOTEDJE 2020). Aussi, faut-il le rappeler, les jeux de coalitions ont sans doute contribué à rehausser les scores

36 En ligne au http://agencecamerounpresse.com/politique/tous-les-résultats-de-l-électionprésidentielle-par-régions.html

${ }^{37}$ Mouvement pour la Renaissance du Cameroun

${ }^{38}$ Social Democratic Front

${ }^{39}$ Mouvement Citoyen National du Cameroun

${ }^{40}$ Union Démocratique du Cameroun

${ }^{41}$ Rassemblement Démocratique du Peuple Camerounais

${ }^{42}$ Alliance pour la Démocratie et le Développement

${ }^{43}$ Peuple Uni pour la Rénovation Sociale

44 Mouvement incluant plusieurs partis appelant au changement «Now», c'est-à-dire maintenant (en anglais), lors de la présidentielle de 2018; son leader est d'origine anglophone. 
des partis politiques et candidats ayant bénéficié des suffrages de leurs alliés pendant cette présidentielle.

Sans être exhaustif, ces allusions montrent à quel point les partis politiques ont des ancrages ethniques et/ou régionaux. Ce qui traduit leur entrain à ne pas perdre au moins leurs contrées d'ancrage qui correspondent en général aux fiefs électoraux, ceci au risque de sceller leur mort politique ou d'hypothéquer leurs espoir de conquérir le pouvoir dans un contexte de compétition pour la survie dans un sens, et pour la conquête du pouvoir dans l'autre.

\section{Conclusion}

Parvenu au terme de notre analyse, il est à retenir selon la littérature consultée que, les activités de transhumance et de pastoralisme au sens étymologique sont liées (RAPPORT FAO, 2015; KONARE \& COULIBALY, 2019). Perçue comme étant un mouvement régulier des peuples pasteurs en direction du sud à la recherche du pâturage (STENNING, 1959 ; CONVERS, 2002 ; CONVERS et al, 2007), la transhumance, dans l'arène politique, contrairement à la définition que lui confère son sens premier, renvoie à la mobilité d'acteur ou groupe d'acteurs quittant temporairement une organisation politique pour une autre avec pour ambition d'y assoupir leur intérêt et revenir au parti politique de départ. En dépit du fait qu'elle soit de la part des acteurs qui en pratiquent, une stratégie de survie dans cet environnement et donc dans le jeu de l'exercice du pouvoir, la transhumance politique donne lieu au changement d'allégeance partisane (POIRIER \& KYELEM, 2012) qui, en étroite liaison avec la transformation de la régulation politique moderne (DUCHASTEL, 2008), est appréhendé comme un fléau pour la démocratie en Afrique. Le fait est qu'il instrumentalise les élus en quête de quelques avantages matériels et de promotion politique. De même, il affaiblit les oppositions dont les élus sont à la merci des majorités au pouvoir, fragilise les équilibres et les contrepoids nécessaires au bon fonctionnement de la démocratie et entretient au finish l'immoralisme et/ ou la mort de certaines forces politiques. (BOUMAKANI, 2008).

Toutefois, un parti politique vivant est celui-là qui est actif. En d'autres termes, c'est une organisation partisane qui compte des militants et sympathisants et qui participe de surcroît aux compétitions politiques. L'analyse des deux modes de mobilité militante, à savoir la transhumance et l'errance politique a permis de mettre en exergue les motivations des acteurs qui y sont impliqués, les stratégies de mobilité qu'ils adoptent et les modes opératoires de survie qu'empruntent les organisations d'opinions soucieuses d'exister en tant que forces politiques. Il se dégage des observations empiriques et des données de sources secondaires que le militantisme transhumant et celui errant prennent corps avec le multipartisme retrouvé. Ces 
pratiques militantes se sont développées et affectent toutes les forces politiques appelées à rivaliser dans l'arène du jeu politique. Pour cela, les forces politiques en présence ne se privent pas de nouer des alliances avec leurs pairs pour gagner en notoriété, conserver la chance d'exister pour certaines ou pour se maintenir en bonne position, conquérir le pouvoir et le conserver pour d'autres. Les jeux de coalition s'érigent ainsi en règles auxquelles presque toutes les forces politiques soucieuses d'exister et de conquérir le pouvoir dans un délai de court, moyen ou long terme adoptent car, pour atteindre ses ambitions tout parti politique a nécessairement besoin d'exister. Quand la perte de ses militants enlevés par la transhumance ou l'errance politique se dresse en contrecourant de ses aspirations politiciennes, il devient impératif d'entreprendre les stratégies d'alliance pour être relevé par les plus imposants.

Le militantisme transhumant et errant constitue une valeur ajoutée pour les formations politiques qui accueillent les membres et sympathisants d'autres organisations d'opinions. Dans ce sens, quand il est arrimé aux jeux de coalitions, il limite les possibilités d'alternance au pouvoir « suprême ». Ceci est d'autant réel parce que le parti au pouvoir, fort de son encrage national qui tend toujours à l'ériger comme un parti «d'état » bénéficie déjà d'un soutien de grande échelle. Quand il obtient en supplément l'allégeance de ses compagnons d'alliance, il va de soi que le maintien du parti au pouvoir devienne inébranlable.

Pour les partis de l'opposition fragilisés pour la plupart par des démissions et abandons de leurs membres militants et sympathisants allés en transhumance ou errant de parti en parti, la survie qui est un impératif ne relève plus seulement des mécanismes classiques de séduction par sa simple existence en tant que parti opposé au régime en place, mais aussi et surtout des pratiques de conscientisation politiques des masses, le développement et le renforcement de la culture politique citoyenne des populations en manque de culture politique portée par des convictions idéologiques.

\section{References:}

\section{Ouvrage}

1. CONVERS, A., CHAIBOU, I., BINOT, A., \& DULIEU, D., (2007), la gestion de la transhumance dans la zone d'influence du parc régional du W. par le programme ECOPAS : une " approche projet » pour l'aménagement de la périphérie du parc, Revue Electronique en Science l'environnement, Hors-Série 4

2. CONVERS, A. (2002), états des lieux spécialisé et quantitatif de la transhumance dans la zone périphérique d'influence du parc National $d u$ W., Crad Emvt

3. NGOH, V., J. (1996), History of Cameroon since 1800, Pressbook. 


\section{Articles et actes de colloques scientifiques}

4. BOUMAKANI, B. (2008). «La prohibition de la transhumance politique des parlementaires. In Étude de cas Africains », Revue française de droit constitutionnel, vol 3, n 75 , pp 499-512

5. BRENAUD, O. (1955). «Nomadisme et transhumance en Afrique subsaharienne, les mouvements pastoraux dans les parcours extrême orientaux du soudan, revue d'élevage et médecine vétérinaire des pays tropicaux », vol4, ${ }^{\circ} 8$, pp327-355

6. CHOUA, B., H. (2001). «Immaturité des politiques et suivie face à la crise économique : les cas de l'Indonésie et de Singapour », revue internationale de politique comparée, vol 8, N³, pp. 473-485

7. GRUENAIS, M., É. \& SCHMITZ, J. (1995). «L'Afrique des pouvoirs et la démocratie », Cahiers d'études africaines, vol 1, n¹37, pp. 7-17

8. ISMAILA DATIDJO \& YOTEDJE, V., D. (2020), « convictions politiques et votes éthno-partisans en périodes électorales au Cameroun à partir du terroir Bamiléké, in politique et économie », cahier de 1'IREA, n 38, pp.89-121

9. KONARE, D., \& COULIBALY, M. (2019), évaluation des impacts de la transhumance sur les ressources pastorales au sud du Mali dans la commune Rurale de Dabia (Cercle de Kéniéba), Europeans Scientific Journal, vol 15, $\mathrm{n}^{\circ} 21, \mathrm{pp} 202-227$

10. MENTHONG, H., L. (1998), «Vote et communautarisme au Cameroun : un vote de cour, de sang et de raison », Politique africaine, vol 3, $\mathrm{N}^{\circ} 69$, PP. 40-52.

11. SINDJOUN, L. (1997), «Élections et politique au Cameroun: concurrence déloyale, coalitions de stabilité hégémonique et politique d'Affection », Afr.j .polit.sci, Vol.2, No.1.

12. TSANA NGUEGANG, R. (2015). «Entrepreneurs-politiciens et populations locales au Cameroun : entre clientélisme et échanges mutuels », In Cahiers d'études africaines, Vol. 4, n² 220, pp.811-835.

13. VAN DE WALLE \& SMIDDY K. (2000), « Partis politiques et systèmes de partis dans les démocraties 'non libérales africaines », In 1'Afrique politique, pp.41-57.

14. ZAMBO BELINGA, J., M. (2007), « la scénographie de la déviance dans les scrutins politiques : la violence comme phénomène électoral d'ici et d'ailleurs », Cahiers d'Etudes Africaines, n¹85, pp.1-30. 


\section{Thèses et Mémoires :}

15. MENGOUEN NCHOUWAT, K. (2017), Transhumance politique à l'Ouest Cameroun: Cas des acteurs politiques de la ville de Foumban, Mémoire de Master en Sociologie, Université de Dschang.

16. NGOH, V., J. (1979), the political evolution of Cameroon 1884-1961, thèse rédigée et soutenue à l'université de Portland.

17. TIENSIGUE, D. (2006), la transhumance politique au Sénégal, Mémoire de Maîtrise en faculté de droit et Science Politique, Université Cheikh Anta Diop.

\section{Rapports :}

18. DUCHASTEL, J. (2008), la modernité politique en question, bulletin $\mathrm{n}^{\circ} 10$ de la chaire de recherche du canada en mondialisation, citoyenneté et démocratie

19. Groupe National du Travail sur l'Élection Présidentielle Sénégalaise, (2010), CODESRIA, Dakar.

20. INS (2011), Annuaire de statistique du Cameroun, Recueil des séries d'informations statistiques sur les activités économiques, sociales, politiques et culturelles du pays jusqu'en 2010 .

21. POIRIER, C. \& KYELEM, B., L. (2012), Nomadisme ou transhumance politique post-électoral et discipline de parti dans l'espace francophone. Assemblée Parlementaire de la Francophonie. Bruxelles (Belgique).

22. Rapport conjointe de mission FA -DRC-CRC (2015), situation de la transhumance et étude socio anthropologique des populations pastorales après la crise de 2014 en République CENTRAFRICAINE (RCA), version finale

\section{Lois :}

23. Loi $\mathrm{n}^{\circ}$ 90/053/ du 19 décembre 1990 portant sur la liberté d'association,

24. Loi ${ }^{\circ}$ 90/056 du 19 Décembre 1990 portant sur la création des partis politiques

\section{Webographie :}

25. AGENCE CAMEROUN PRESSE, http://agencecamerounpresse.com/politique/tous-les-résultats-de-lélection-présidentielle-par-régions.html

26. Archive des résultats des élections parlementaires au Cameroun sur : https://www.archive.ipu.org/parline-f/reports/1053_arc.htm

27. ESSOGO, A. (2020), «Jean Jacques EKINDI rentre au RDPC», in Cameroon-tribune, sur https://www.cameroon-tribune.cm/article;html/32167/fr.html/jeanjacques-ekindi-rentre-au-rdpc. Publié le 04 mai 2020. 
28. FOUTE, F. (2020), «au Cameroun, les premières leçons d'un scrutin boudé par les électeurs », in Jeune-afrique, sur https://www.jeuneafrique.com/894867/politique/au-cameroun-lespremières-leçons-dun-scrutin-boude-par-les-citoyens/. Publié en ligne le 11 février 2020.

29. MAMAN, J. (2018), «les alliés du RDPC en bonne place », in Cameroon-tribune, sur https://www.cameroon-tribune.cm/article.html/21002/fr.html/alliésdu-rdpc-en-bonne-place. publié en ligne sur le 01 octobre 2018.

30. SEHOU, A. (2020), «Les autorités administratives sont des entraves à l'expression démocratiques », sur www.leseptentrion. net. 